\title{
A General Data Grid: Framework and Implementation
}

\author{
Wu Zhang, Jian Mei, and Jiang Xie \\ Department of Computer Science and Technology, Shanghai University, \\ Shanghai, 200072, China \\ zhang@mail.shu.edu.cn, meijian_2003@yahoo.com.cn
}

\begin{abstract}
Today, data grids have become an important emerging platform for managing and processing a very large amount of data distributed across multiple grid nodes and stored in relational databases. However, there are still obstacles for potential grid users to be involved into the trend and the data grid application development is far from the data grids. While the traditional established data grid architectures are not particularly suitable for the some grid service, such as data replica services, user authentication services, and the Optimal Path selection services. Our project aims to exploit a novel architecture named General Data Grid, which integrates the metadata services, data replica services, java message services, and the Optimal Path selection on Data Grid environment. At the end of this paper, we describe the key implement on the GDGrid and present a simple example application concerning finding the optimal route. Our experiment of GDGrid shows the algorithm of the route selection (Heart-Beat algorithm) is effectively, improve the performance greatly, and afford fault-tolerance management to the great extent.
\end{abstract}

\section{Introduction and Relate Work}

With the emergence of the massive data collections of terabyte scale and the highperformance computers, how to utilize these huge data sets and the high-performance computing capacity becomes a great challenge.

Under the circumstances, "Grid" is appearing as are an approach for building dynamically constructed problem-solving environments using geographically and organizationally dispersed high-performance computing and data-handling resources [1]. Thus today can be used as effective tools for distributed computing and data processing in many domains such as astronomy, geography, and earthquake, etc. In all the Grid applications, Data Grid plays an important role in the data handling. It is a dynamic logical namespace that enables coordinated sharing of heterogeneous distributed storage resources and digital entities based on local and global policies across administrative domains in a virtual enterprise[2].

In the Architecture, European Data Grid Project [3] is the development of a new environment to support globally distributed scientific exploration involving terabytes datasets. And the GriPhyN Project [4] provides a new degree of transparency in how data-handling and processing capabilities are integrated to deliver data products to end-users or applications. These two projects are strongly related to grid services architecture, but they do not address more sophisticated planners, which can take into 
account performance and reliability as well as provide feedback to the user, so that the user can decide whether to go ahead with a request. In the data replicas management, a simulation framework - OptorSim was introduced in [5] where data replication was combined with job scheduling. They uses a prediction function based on spatial and time locality regardless of the overall data access cost on the Data Grid.

In this paper we propose a novel framework for access control, message services, data replicas services and the selection of the OP (Optimal Path) in Data Grid environment. Our key contributions include the following aspects: (1) JMS (Java Message Service) is employed on subscribe/cancel service, replicas service, and Heart-Beat service. Through the combine of the GridFTP and JMS, we can ensure the replicas' consistency and validity. (2) In the access control management, we introduce the mechanism of inspection certificates. This module is responsible for verifying the certificates when the users visit the specified access binaries. (3) In the selection of the OP (Optimal Path), we employ the Heart-Beat algorithm. By means of this algorithm, we can find the temporal OP with a little expense.

The rest of the paper is organized as follows. In Section 2, we describe the related work. We describe the general data grid framework in detail in Section 3. In Section 4, we show the key implementation status of the general data grid and Section 5 concludes the paper and discusses future work.

\section{The General Data Grid Framework}

The GDGrid (General Data Grid) is built on the OGSA-DAI [6]. We present a framework of GDGrid as Fig.1 shows. The GDGrid is composed of three Layers, which are the SRL (Service Requesting Layer), the SML (Service Managing Layer) and the SPL (Service Providing Layer) [7].

\subsection{SRL (Service Requesting Layer)}

In the SRL, the SC (Service Client) can visit the SWP (Service Web Portal) of the GDGrid. Through the SWP, the SC can register the own data services into the GDGrid and become a SP (Service Provider). The SC can attain the service provided by the GDGrid without the protected data resources in the SWP. In case that the SC wants to get the services of the protected data resources, it must log on the AM (Access Management) module and get the relevant certificates of the protected data resources. In the GDGrid, once the SC would like to become a SP, it must download the Middleware of the SPL (Service Providing Layer) and perform other operations what we will discuss in the following sections. In this layer, the SC will interact with the SM (Scheduling Management) module. The SC advances a request, and then the SM will offer the temporal optimal route according to the heart-beat algorithm to the SC.

\subsection{SML (Service Managing Layer)}

The Service Managing Layer is the core of the GDGrid. Its main goals are to furnish the data grid interface to the SCs, integrate the heterogeneous data, offer a secure environment and find the Optimal Path of the request execution. Moreover, this layer 


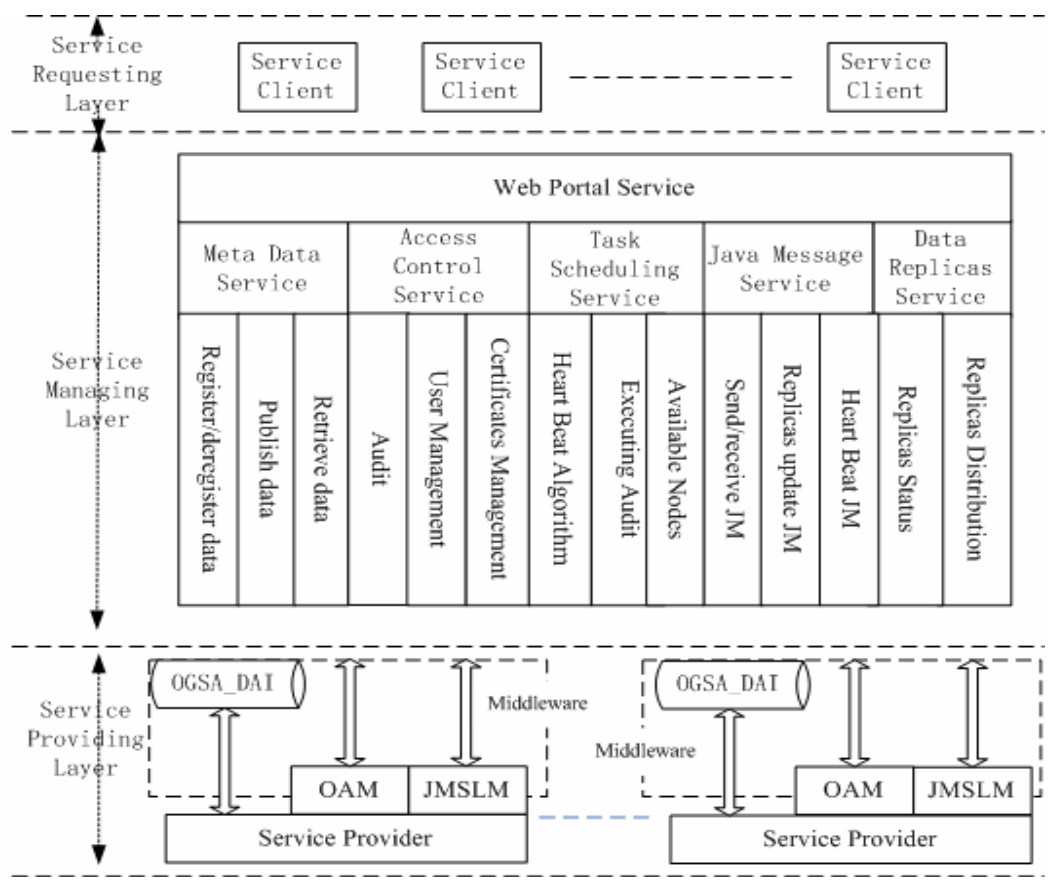

Fig. 1. The GDGrid Architecture

coordinates the application execution by attempting to fulfill the application requirements and the available data resources. On the whole, this layer consists of six main services.

The WPS (Web Portal Service) builds the relationship between the SRL and the SPL. In the GDGrid, the WPS is responsible for maintaining a description of the GDGrid. In the other words, we can think it as an interface of the GDGrid. To be the $\mathrm{SC}$, it can log in the Data Grid, obtain the GDGrid's services freely and register its services to be a SP. To be the SP, it can register or deregister its services and the descriptions, change its service, obtain the GDGrid's services, request a certificate of its own, and authorize its own certificate replicas to the other dependable SP, etc.

The MDS (Meta Data Service) offer all the metadata describing features of data sources, and maintain the catalogue of the original data and the catalogue of replicas data. The ACS (Access Control Service) focuses are on managing the certificates, empowering the users to utilize the data resources and presenting the monitoring of the logged users. In the monitoring management, we make use of a web based monitoring tool (Map Center [8]) which provides access to status information.

The JMS (Java Message Service) offer three kinds of service. One service aims to send the real-time messages to any other nodes, and the second service is used to customize the some services, such as getting the latest public information, the state of the original data and so on. The last service is the Heart-Beat message service.

The DRS (Data Replicas Service) is used to coordinate data resources replication across the GDGrid from one node to another. There are including three parts: Replicas Catalog, Replicas States and Replicas Descriptions. 
In the TSS(Task Scheduling Service), we propose an algorithm called Heart-Beat, which is the SPs (Service Providers) send some key own information [11] (such as processor speed, memory size, and I/O performance, available bandwidth) to the TSS module at regular intervals.

We make an assumption that the job is divided into $N$ subjobs, and at that time $M$ nodes are available. In the GDGrid, we calculate the job execution time through the Formula $1 . P_{j}$ is the performance of computer j. Time $e_{\text {Exec } \_i j}$ is the expected execution time of the subjob i on the Node j. $S_{\text {Input } j}, S_{\text {Application } j \text { j }}$ and $S_{\text {output } \_j}$ represent the size of the input data, the application code and the output data of the subjob $\mathrm{j}$ respectively. $W_{j}$ is the bandwidth of the Node j. $P_{j}$ is the performance parameters of the Node $\mathrm{j}$. and $T_{j}$ refer to the available system resource of Node $\mathrm{j}$ such as the throughput of I/O.

$$
\begin{aligned}
& \text { Time }_{\text {Re } \text { sponse }_{-} j}=\text { Time }_{\text {arrived }_{-} j}-\text { Time }_{\text {send }_{-} j} \\
& \text { Time }_{\text {Exec_ij }}=\left(\frac{S_{\text {Input_i }_{-}}+S_{\text {Application_i }_{-}}}{W_{j} \times P_{j} \times T_{j}}+\frac{S_{\text {Output_i }_{-}}}{W_{j} \times P_{j}}\right) \times \text { Time }_{{\mathrm{Re} s p o n s e_{-} j}}
\end{aligned}
$$

The results obtained from the Formula 1 can be used for the construction of an $\mathrm{M} \times \mathrm{N}$ matrix. Through the following algorithm, we can find the optimal quickly and accurately.

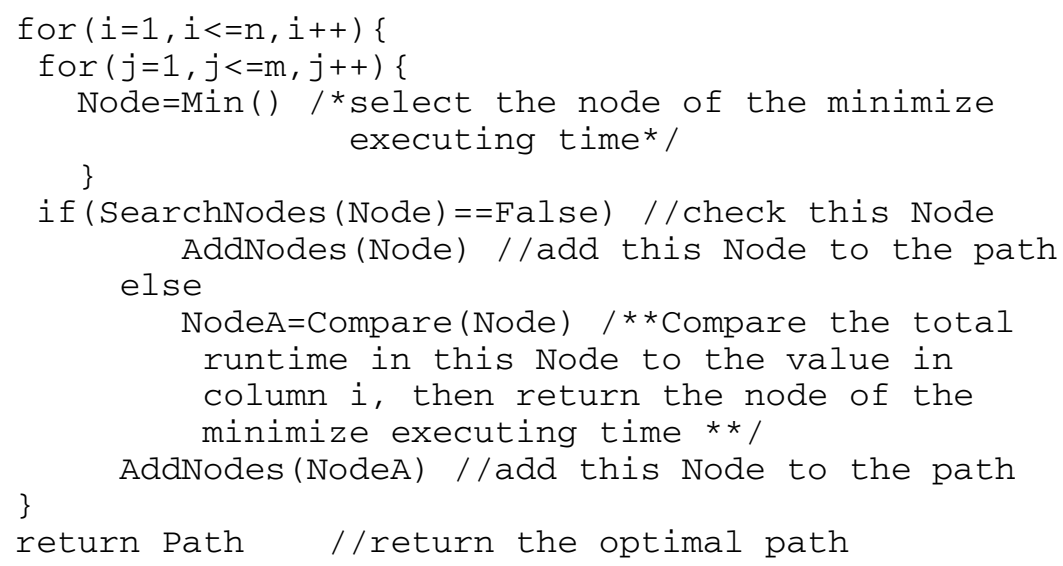

\subsection{SPL (Service Providing Layer)}

The SRL is basically used for organizing the SPs (Service Providers) orderly, utilizing the SPs' resource efficiently and administering the certificates securely. In the SRL, the OGSA-DAI are applied to integrate various heterogeneous data resources seamlessly and to build the relationship between the SPs and the SML.

As for a SP, JMSLM (Java Message Service Local Module) is used to receive the input JM issued by other SPs and to send the output JM to the destination. In this GDGrid, the input JM can be considered a manner which the other SPs notify the current SP. We assume that a current SP holds a replica of a remote SP's data resource. Once the remote SP's data resource is changed, the remote SP will send a notice of the changes to the JMS of the SML through the JMSLM, the JMS will distribute the notice 
to all the nodes which possess the replicas, and finally the JMSLM will proceed with the notice in the SP. As for the remote SP the notice is an output JM, while as for the current SP the notice is an input JM. In addition, the Heart-Beat is considered as an output JM for a SP and send through the JMSLM in the same way.

In the SP, there is another module named OAM (Own Authentication Module) which is adopted with the purpose of recording the own and other certificates information.

\section{The General Data Grid Key Implementation}

\subsection{Java Message Service}

Java message service is implemented based on the JMS of the SML and the JMSLM of the SPL. It includes three major message services: the subscribe/cancel service, the replicas service, and the Heart-Beat service.

During the course of designing the JMS, the advanced EJB technology and the design patterns are employed to the GDGrid. If the SCs have interest in some SPs, they can subscribe the latest information of some SPs by the Subscribe Message service. In case that the SPs accept the subscribe request, the SPs will send to the realtime latest information to the SCs timely. If the SCs have no interest in the SPs any more, they can cancel the order by the Cancel Message service.

The Replicas Message service associating with the GridFTP makes it possible that the data replicas can keep the consistency and validity. Once a SP's data resource is modified, the SP will send a notice of the changes to the Replicas Message service, it will distribute the notice to all the nodes possessing the replicas. If a node possessing a replica is closed when the original data are changed, the Replicas Message service will put the notice to the massage cache area. Once the node is available, the Replicas Message service will resend the notice to the node. In the management of the massage cache area, GDGrid provides the service mechanism called Least Recently Used (LRU). That is the node which is accessible in the first time owns the headmost services and receives the message about itself.

As for the Heart-Beat message service, it plays a vital role to the selection of the optimal path. Through the service, the GDGrid can attain the correct real-time information about the usable nodes.

\subsection{Authorization Service}

About the authorization service, we can explain through the Fig.2.

Step 1: The user A sends a request for owning an own unique certificate in order to protect some protected data resources.

Step 2: The ACS module assigns a unique certificate (For example CTF001, X509) for the protected data resources of user A. Once other users want to visit those data resources, they should own the CTF001.

Step 3: User B sends a request for visiting the protected data resources of user A. 
Step 4: The ACS module transmits the request to the User A. The request consists of the information of the demander (User B) username, IP address, the position etc.

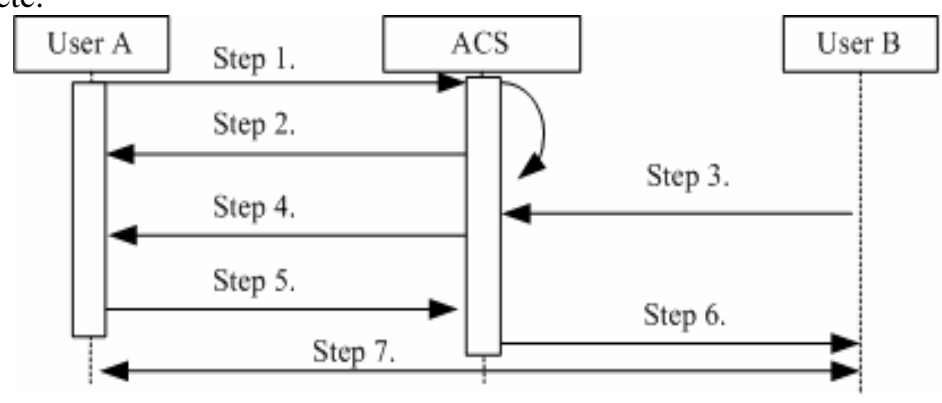

Fig. 2. Procedure In Authorization

Step 5: The User A confirms the request and returns a message (yes or no). If the message is no, the ACS module will refuse the request which is send by the demander. In the Fig.2, the User A sends the message which is yes.

Step 6: The ACS module receives the message form User B, checks the certificates management sub module, and sends a replica of the User A's certificate to the User B.

Step 7: Once the User B owns the replica of the User A's certificate, he can use the User A data resources directly without through the ACS module certification.

By this means, we not only save the authentication time and the bandwidth, but also make utmost use of the data resources in the safe situation.

\subsection{The Optimal Path Discovery}

Experiments are taken to analyze the process of finding the optimal path. The job is divided into four subjobs and there are six accessible and available nodes at the experiments. The parameters used in the experiments are list as follows: $\angle C P U$, Memory, Bandwidth, Available_Resouce, Response_Time $>,\{$ Node_1 $<1 \mathrm{G}, 256 \mathrm{M}$, $1.85 \mathrm{M}, 80 \%, 50 \mathrm{~S}>$, Node_2<2G, 512M, 1.7M, 100\%, 45S $>$,Node_3<2G, 512M, $1.6 \mathrm{M}, 80 \%, 45 \mathrm{~S}>$,Node_4<2.5G, 512M, 17M, 70\%, 45S >,Node_5<2G 2G, $1 \mathrm{G}$, $1.8 \mathrm{M}, 55 \%, 45 \mathrm{~S}>$,Node_6 $<2.6 \mathrm{G}, 512 \mathrm{M}, 1.74 \mathrm{M}, 60 \%, 50 \mathrm{~S}>$ \}.

Table 1. The forcase of executing subjob. the Zero means the node has the subjob's input data.

\begin{tabular}{|l|l|l|l|l|l|l|l|l|l|l|l|l|l|}
\hline \multirow{2}{*}{ Nodes } & \multicolumn{1}{|l|}{ Forecast Subjob1 } & \multicolumn{3}{|l|}{ Forecast Subjob2 } & \multicolumn{3}{l|}{ Forecast Subjob3 } & \multicolumn{3}{|c|}{ Forecast Subjob4 } \\
\cline { 2 - 14 } & S_in & S_ap & S_ou & S_in & S_ap & S_ou & S_in & S_ap & S_ou & S_in & S_ap & S_ou \\
\hline Node_1 & $30 \mathrm{M}$ & $10 \mathrm{M}$ & $12 \mathrm{M}$ & $25 \mathrm{M}$ & $15 \mathrm{M}$ & $10 \mathrm{M}$ & $0 \mathrm{M}$ & $8 \mathrm{M}$ & $8 \mathrm{M}$ & $12 \mathrm{M}$ & $10 \mathrm{M}$ & $10 \mathrm{M}$ \\
\hline Node_2 & $30 \mathrm{M}$ & $10 \mathrm{M}$ & $12 \mathrm{M}$ & $25 \mathrm{M}$ & $15 \mathrm{M}$ & $10 \mathrm{M}$ & $15 \mathrm{M}$ & $8 \mathrm{M}$ & $8 \mathrm{M}$ & $12 \mathrm{M}$ & $10 \mathrm{M}$ & $10 \mathrm{M}$ \\
\hline Node_3 & $0 \mathrm{M}$ & $10 \mathrm{M}$ & $12 \mathrm{M}$ & $25 \mathrm{M}$ & $15 \mathrm{M}$ & $10 \mathrm{M}$ & $15 \mathrm{M}$ & $8 \mathrm{M}$ & $8 \mathrm{M}$ & $0 \mathrm{M}$ & $10 \mathrm{M}$ & $10 \mathrm{M}$ \\
\hline Node_4 & $30 \mathrm{M}$ & $10 \mathrm{M}$ & $12 \mathrm{M}$ & $25 \mathrm{M}$ & $15 \mathrm{M}$ & $10 \mathrm{M}$ & $15 \mathrm{M}$ & $8 \mathrm{M}$ & $8 \mathrm{M}$ & $12 \mathrm{M}$ & $10 \mathrm{M}$ & $10 \mathrm{M}$ \\
\hline Node_5 & $30 \mathrm{M}$ & $10 \mathrm{M}$ & $12 \mathrm{M}$ & $0 \mathrm{M}$ & $15 \mathrm{M}$ & $10 \mathrm{M}$ & $15 \mathrm{M}$ & $8 \mathrm{M}$ & $8 \mathrm{M}$ & $12 \mathrm{M}$ & $10 \mathrm{M}$ & $10 \mathrm{M}$ \\
\hline Node_6 & $30 \mathrm{M}$ & $10 \mathrm{M}$ & $12 \mathrm{M}$ & $25 \mathrm{M}$ & $15 \mathrm{M}$ & $10 \mathrm{M}$ & $15 \mathrm{M}$ & $8 \mathrm{M}$ & $8 \mathrm{M}$ & $12 \mathrm{M}$ & $10 \mathrm{M}$ & $10 \mathrm{M}$ \\
\hline
\end{tabular}


According to calculating these parameters through the Formula 1, the $6 \times 4$ matrix is shown as follows:

\begin{tabular}{|c|c|c|c|c|c|c|c|c|}
\hline & Subjobl & Subjob2 & Subjob3 & Subjob4 & Subjob1 & Subjob2 & Subjob3 & Subjob4 \\
\hline Node_1 & {$[1675.7$} & 1621.6 & 486.5 & 1013.57 & {$[1675.7$} & 1621.6 & 486.5 & 1013.5 \\
\hline Node_2 & 611.8 & 588.2 & 364.7 & 376.5 & 258.8 & 294.1 & 364.7 & 235.3 \\
\hline Node_3 & 344.5 & 843.7 & 516.8 & 316.4 & 344.5 & 404.3 & 516.8 & 316.4 \\
\hline Node_4 & 650.8 & 631.9 & 384.5 & 389.9 & 650.8 & 631.9 & 384.5 & 389.9 \\
\hline Node_5 & 528.5 & 233.0 & 311.4 & 312.5 & 528.5 & 233.0 & 311.4 & 312.5 \\
\hline Node_6 & 753.5 & 734.4 & 443.8 & 447.0 & 753.5 & 734.4 & 204.3 & 447.0 \\
\hline
\end{tabular}

Fig. 3. The Execution Time. Left matrix is without data replicas, and the right is with replicas.

When choosing the optimal path, the SM will use the Heart-Beat algorithm. In this experiment without data replicas, the optimal path is Node_3 $\rightarrow$ Node_5$\rightarrow$ Node_2 $\rightarrow$ Node_4. Also another experiment is done during the period of executing this job, we shut down the Node_2. The SM will process with the adaptive executing path choice. That is in this experiment the subjob_3 executed on the Node_2. When the Node_2 is inaccessible, the Heart-Beat algorithm will select the optimal node to execute the subjob_3 expect the Node_2. So the optimal path is changed to be Node_3 $\rightarrow$ Node_5 $\rightarrow$ Node_l $\rightarrow$ Node_4 in this experiment. Through this experiment, it proves that the Heart-Beat algorithm is effectively, improve the performance greatly, and afford fault-tolerance management to some extent.

For the purpose of finding the data replicas' effect on the scheduling, we make some data replicas on the different nodes (in this experiment, the time of the replicas' transfer is not considered.): employ the subjob1's data on the Node_2 and Node_3; the subjob2's data on Node_2, Node_3 and Node_5; the subjob3's data on Node_1 and Node_6; the subjob4's data on Node_2 and Node_3. By using the Formula 1, the execution time with data replicas $6 \times 4$ matrix is gotten, and the optimal path is: Node_2 $\rightarrow$ Node_5 $\rightarrow$ Node_6 $\rightarrow$ Node_3.

From the two $6 \times 4$ matrixes, we can figure out the probable total runtime. One with no data replica is $389.9 \mathrm{~s}$, and the other with some data replicas is $316.4 \mathrm{~s}$. Obviously, the data replicas must short the runtime of the task.

\section{Conclusion and Future Work}

The GDGrid facilitates the usability of the distributed heterogeneous data resources on the Grid. It integrates Metadata services, Data Replica services, Java Message services, Replicas management services and route selection within its framework. In this paper, we have described the three level of the GDGrid in detail and introduce the functions of different sub-modules briefly. We present the key implementations and do some experiments that validate the efficiency of our Heart-Beat algorithms.

Additional future work of this project will be to integrate and classify data resources. In addition, to extend the performance of data transfers, we intend to investigate protocols based on Quality of Service concerning the transformation of large quantities of data. Further, since in our GDGrid the dynamic transfer of the data 
replicas is considered in the optimal path selection, we plan to add this consideration into the Heart-Beat algorithm and improve the algorithm in the efficiency and speed.

\section{References}

1. I.Foster and C.Kesselman (Eds): The Grid: Blueprint for a New Computing Infrastructure. http://www.mkp.com/books_catalog/1-55860-475-8.asp, Morgan Kanfmann, Los Altos, CA,1988.

2. Reagan.Moore, Arcot rajasekar, and Michal Wan, MEMBER, IEEE: Data Grids, Digital Libraries, and Persistent Archives: An Integrated Approach to Sharing, Publishing, and Archiving Data. Proceedings of the IEEE, VOL. 93, NO. 3, 578-588, March 2005.

3. Segal, B.; Robertson, L.; Gagliardi, F.; Carminati, F.: Grid computing: the European Data Grid Project. Nuclear Science Symposium Conference Record, 2000 IEEE Volume 1, 1520 Oct. 2000 Page(s):2/1 vol.1

4. Deelman, E., Kesselman, C., Mehta, G., Meshkat, L.: GriPhyN and LIGO, building a virtual data Grid for gravitational wave scientists. High Performance Distributed Computing, 2002. HPDC-11 2002. Proceedings. 11th IEEE International Symposium on Page(s):225 - 234

5. William H. Bell, David G. Cameron, Luigi Capozza, A. Paul Millar, Kurt Stockinger, Floriano Zini: Simulation of Dynamic Grid Replication Strategies in OptorSim. Proc. Of the 3rd Int'1 IEEE workshop on Grid Computing (Grid 2002), Baltimore, USA.

6. Open Grid Services Architecture - Data Access and Integration Project: http://www.ogsadai.org.uk.

7. Nong Xiao, Dongsheng Li, Wei Fu, Bin Huang, Xicheng Lu: GridDaen: A Data Grid Engine. Second International Workshop,GCC2003, Shanghai, China, Page(s):519-528

8. Map Center Home Page: Http://mapcenter.in2p3.fr

9. G. Aloisio, M. Cafaro, I. Epicoco: Early experiences with the GridFTP protocol using the GRB-GSIFTP library. Future Generation Computer Systems, Volume 18, Number 8 (2002), pp. 1053-1059, Special Issue on Grid Computing: Towards a New Computing Infrastructure, North-Holland.

10. Ann Chervenak, Ian Foster, Carl Kesselman, Charles Salisbury and Steven Tuecke: The Data Grid: Towards an Architecture for the distributed Management and Analysis of Large Scientific Datasets. Journal of Network and Computer Applications, 23:187-200, 2001.

11. Sang-Min Park, Jai-Hoon Kim: Chameleon: A Resource Scheduler in A Data Grid Environment*. Cluster Computing and the Grid, 2003. Proceedings. CCGrid 2003.3rd IEEE/ACM International Symposium on12-15 May 2003 Page(s):258 - 265. 